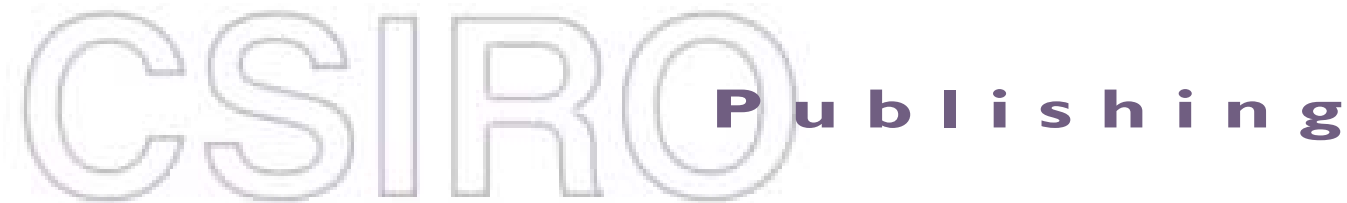

\section{Publications of the Astronomical Society of Australia}

Volume 19, 2002

(C) Astronomical Society of Australia 2002

An international journal of astronomy and astrophysics

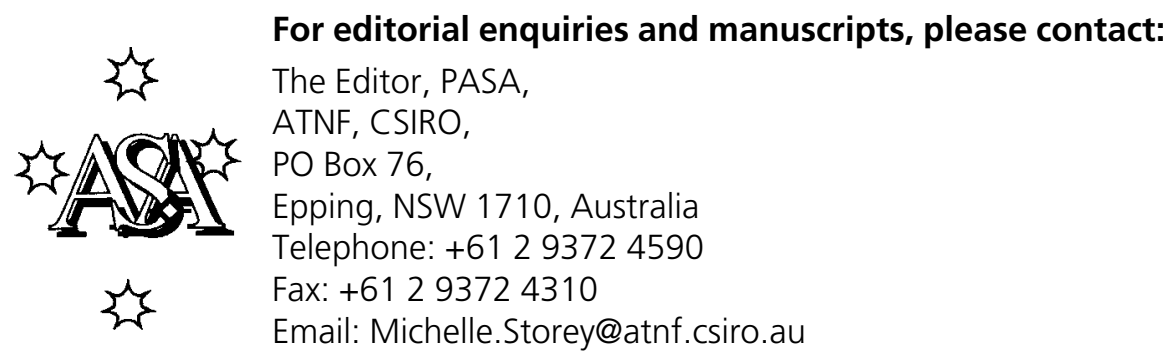

For general enquiries and subscriptions, please contact: CSIRO Publishing PO Box 1139 (150 Oxford St)

Collingwood, Vic. 3066, Australia

Telephone: +6139662 7666

Fax: +61 396627555

Email: publishing.pasa@csiro.au

C S I RO

PUBLISHING Published by CSIRO Publishing

for the Astronomical Society of Australia

www.publish.csiro.au/journals/pasa 


\title{
A Catalogue of Damped Lyman Alpha Absorption Systems and Radio Flux Densities of the Background Quasars
}

\author{
S. J. Curran ${ }^{1}$, J. K. Webb ${ }^{1}$, M. T. Murphy ${ }^{1}$, R. Bandiera ${ }^{2}$, \\ E. Corbelli ${ }^{2}$ and V. V. Flambaum ${ }^{1}$ \\ ${ }^{1}$ School of Physics, University of New South Wales, Sydney NSW 2052, Australia \\ sjc@bat.phys.unsw.edu.au \\ ${ }^{2}$ Osservatorio Astrofisico di Arcetri, Largo E. Fermi, 5, 50125, Italy \\ Received 2001 September 21, accepted 2002 June 25
}

\begin{abstract}
We present a catalogue of the 322 damped Lyman alpha absorbers taken from the literature. All damped Lyman alpha absorbers are included, with no selection on redshift or quasar magnitude. Of these, 123 are candidates and await confirmation using high resolution spectroscopy. For all 322 objects we catalogue the radio properties of the background quasars, where known. Around 60 quasars have radio flux densities above $0.1 \mathrm{Jy}$ and approximately half of these have optical magnitudes brighter than $V=18$. This compilation should prove useful in several areas of extragalactic/cosmological research.
\end{abstract}

Keywords: catalogues — quasars: absorption lines — radio continuum: galaxies — early universe

\section{Introduction}

High resolution spectroscopy of quasars reveals large numbers of absorption lines due to neutral atomic hydrogen. In some cases, the neutral hydrogen column density can be very large $\left(N_{\mathrm{HI}} \gtrsim 10^{20} \mathrm{~cm}^{-2}\right)^{*}$ and gives rise to a heavily damped absorption feature (e.g. Wolfe et al. 1986). The aim of this paper is to present a catalogue of these damped Lyman alpha absorption systems (DLAs) and, where known, the radio flux densities of the background quasar. The catalogue has been compiled from the large number of studies reported in the literature.

The nature of DLAs is still open to debate. Interpretations include galactic disks (Wolfe et al. 1986), low surface brightness galaxies (Jimenez, Bowen \& Matteucci 1999) or dwarf galaxies (Matteucci, Molaro \& Vladilo 1997) intersecting the sight-line towards a background quasar. Despite these possible differing morphologies, DLAs provide a powerful cosmological probe. The highest redshift $(z>3.5)$ DLAs may account for a large fraction of the baryons at high redshift, suggesting they reveal gas prior to the bulk of the star formation history of the universe (Péroux et al. 2002, and references therein). On the other hand, recent work (Lanzetta et al. 2002) seems to indicate star formation rates which continue to increase with increasing redshift up to the highest galaxy redshifts observed in the Hubble Deep Field. The discovery of a DLA with truly primordial abundances would have a major impact on our understanding of the early chemical evolution of the universe, and a crucial reality check on the ever-elusive population III. This will also be important for studies of primordial deuterium abundances (see below),

\footnotetext{
*In the catalogue some column densities less than this are to be found. This is because in order to produce the most comprehensive list, we have also included any Lyman alpha absorbers which are designated as possibly damped in the literature (see Section 2).
}

since deuterium is destroyed, and never created, by star formation and evolution.

High resolution spectroscopy can be used to study high chemical abundances over a large redshift range. In particular, the difficult ionisation corrections required to derive meaningful chemical abundances in Lymanlimit absorbers (where $\log N_{\mathrm{HI}}>17.2 \mathrm{~cm}^{-2}$, so that they are optically thick to Lyman continuum radiation, e.g. Lanzetta 1991) can be avoided using DLAs since the observed hydrogen is probably all neutral (Turnshek et al. 1989; Lanzetta et al. 1991). Additionally, at high neutral hydrogen column densities, species such as $\mathrm{Zn}$ II and $\mathrm{Cr}$ II may become detectable, which are important since depletion onto dust grains is thought to be negligible for the former, whereas the latter remains in the solid phase. This allows both the study of abundances and depletion patterns/dust reddening (see Pettini, Boksenberg \& Hunstead 1990; Pettini et al. 1997). Some further reasons why DLAs are of interest are:

1. Studies of the higher order hydrogen Lyman series in DLAs can be used to investigate the primordial deuterium abundance (Webb et al. 1991). The advantage of using DLAs is that the deuterium column density can be somewhat larger than typical Lyman forest absorbers. This may help to discriminate against $\mathrm{HI}_{\mathrm{I}}$ interlopers mimicking the deuterium line. Two recent observational studies (D'Odorico, DessaugesZavadsky, \& Molaro 2001; Pettini \& Bowen 2001) report such D/H measurements.

2. Radio observations of quasars with a sufficiently high radio flux density can provide information complementary to that of the DLA observations: $21 \mathrm{~cm} \mathrm{H} \mathrm{I}$ measurements reveal more detailed kinematic information since line saturation is less severe and provide a direct spin temperature of the cool component of the gas. Different radio and optical morphology of the 
background quasar also provides the opportunity of observing along slightly different sight-lines through the same absorption complex, with the potential of learning about the relative sizes of optical/radio emission regions and the cloud size of the absorbing gas. In those rare cases where the host quasar has a sufficiently strong millimetre flux and a foreground molecular cloud occults the quasar (Wiklind \& Combes 1994a; Wiklind \& Combes 1996), a wealth of detailed chemistry is revealed (Gerin et al. 1997; Combes \& Wiklind 1999).

3. Studies of high redshift dust in DLAs gives a handle on the chemical evolution and star formation rates at various cosmological epochs (Pei, Fall \& Hauser 1999) through the contribution of dust to quasar spectral energy distributions (e.g. Klein et al. 1996; Bertoldi et al. 2000; Carilli et al. 2000; Omont et al. 2001).

4. Certain heavy element transitions provide cosmological probes of special interest. For example, species with ground and excited state transitions sufficiently close to each other in energy provide a unique means of measuring the cosmic microwave background temperature at high redshift (Bahcall, Joss, \& Lynds 1973; Meyer et al. 1986; Srianand, Petitjean, \& Ledoux 2000).

5. Finally, recent detailed studies of the relative positions of heavy element atomic optical transitions and comparison with present day (laboratory) wavelengths, suggest that the fine-structure constant $\left(\alpha \equiv e^{2} / \hbar c\right)$ may have evolved with time. Inter-comparing atomic optical transitions with $\mathrm{H}_{\mathrm{I}} 21 \mathrm{~cm}$ and molecular millimetre transitions may yield an order of magnitude over the already highly sensitive optical results (Cowie \& Songaila 1995; Drinkwater et al. 1998; Murphy et al. 2001b). However, very few such constraints are available due to the paucity of quasar absorption systems where 2 of the 3 types of transition (optical atomic, $\mathrm{H}$ I $21 \mathrm{~cm}$ or molecular millimetre) exist.

It is this last point which is of interest to us: as well as providing a comprehensive list of these objects for use by the astronomical community in general (Section 2), this catalogue allows us to shortlist those DLAs most likely to exhibit radio absorption lines in order to further constrain the variation in the fine-structure constant. In the final sections we present the DLAs occulting radio-loud quasars along with any radio absorption features published and outline our future plans regarding the sample.

\section{Explanatory Comments and References}

In Table 1, for the column density we note that Corbelli, Salpeter \& Bandiera (2001) argue that when $N_{\mathrm{HI}}$ is estimated directly from the absorption line equivalent width, the value is systematically underestimated when compared to the estimate derived using Voigt profile fitting. This may be due to a bias associated with estimating the quasar continuum due the presence of Lyman forest absorption lines. The values of $N_{\mathrm{HI}}$ quoted in the table in this paper are those values reported in the original sources, and are not corrected using the relation given in Corbelli et al. (2001).

Note also that the visual magnitude is obtained from the DLA reference, Véron-Cetty \& Véron (2001) or, failing these, the NASA/IPAC Extragalactic Database (NED), which gives an approximate value. For the radio flux densities, $S_{0.4}$ is either the $0.33 \mathrm{GHz}$ WENSS, $0.37 \mathrm{GHz}$ Texas or $0.41 \mathrm{GHz}$ MRC flux density (see Section 2.2), and where both flux densities are available the $365 \mathrm{MHz}$ value is quoted. In the case of the Texas survey, ' $\mathrm{X}$ ' denotes that the quasar was not detected at the flux density limit of $0.25 \mathrm{Jy}$ (Douglas et al. 1992). $S_{1.4}$, etc., are the measured $1.4 \mathrm{GHz}$, etc., continuum flux densities in $\mathrm{Jy}$, and for $S_{\text {higher }}$, the frequency in $\mathrm{GHz}$ is given in parentheses. In the appropriate columns ' $\mathrm{X}$ ' also designates if the object is not considered a 2.7 or $5.0 \mathrm{GHz}$ radio source (VéronCetty \& Véron 2001), and where the 22 and $37 \mathrm{GHz}$ values are quoted as approximate refers to the average value from 15 years of monitoring by Teräsranta et al. (1998). In general, approximate values are used when the flux density is obtained from more than one reference and the values given do not exactly agree. Finally, '-' denotes that no information could be found.

\subsection{The DLA References}

The DLAs are compiled from Wolfe et al. (1978) ${ }^{1}$, Wolfe \& Davis $(1979)^{2}$, Wolfe, Briggs \& Jauncey $(1981)^{3}$, Snijders et al. (1982) ${ }^{4}$, Tytler $(1982)^{5}$, Bechtold et al. $(1984)^{6}$, Wolfe et al. (1986) ${ }^{7}$, Black, Chaffee \& Foltz $(1987)^{8}$, Tytler $(1987 a)^{9}$, Tytler $(1987 b)^{10}$, Lanzetta $(1988)^{11}$, Sargent, Steidel \& Boksenberg $(1989)^{12}$, Turnshek et al. (1989) ${ }^{13}$, Lanzetta $(1991)^{14}$, Lanzetta et al. $(1991)^{15}$, Schneider, Schmidt \& Gunn $(1991)^{16}$, Beaver, Cohen \& Junkkarinen (1992) ${ }^{17}$, Courvoisier \& Paltani $(1992)^{18}$, Meyer \& York (1992) ${ }^{19}$, Bahcall et al. $(1993)^{20}$, Lu et al. $(1993)^{21}$, Turnshek \& Bohlin $(1993)^{22}$, White, Kinney \& Becker $(1993)^{23}$, Chaffee et al. (1994) ${ }^{24}$, Wolfe et al. $(1994)^{25}$, Lu et al. $(1995)^{26}$, Lanzetta, Wolfe \& Turnshek $(1995)^{27}$, Steidel et al. $(1995)^{28}$, Wolfe et al. $(1995)^{29}$, Bahcall et al. $(1996)^{30}$, Carilli et al. $(1996)^{31}$, de Bruyn, O'Dea \& Baum (1996) ${ }^{32}$, Impey et al. $(1996)^{33}$, Lu et al. (1996) ${ }^{34}$, Petitjean et al. (1996) $)^{35}$, Stepanian et al. $(1996)^{36}$, Storrie-Lombardi et al. (1996) ${ }^{37}$, Ge \& Bechtold $(1997)^{38}$, Lanzetta et al. (1997) ${ }^{39}$, Le Brun et al. $(1997)^{40}$, Lu, Sargent \& Barlow $(1997)^{41}$, Vladilo et al. $(1997)^{42}$, Ivison, Harrison \& Coulson $(1998)^{43}$, Jannuzi et al. $(1998)^{44}$, Le Brun, Viton \& Milliard $(1998)^{45}$, Srianand \& Petitjean (1998) ${ }^{46}$, Leibundgut \& Robertson $(1999)^{47}$, de la Varga et al. $(2000)^{48}$, Molaro et al. $(2000)^{49}$, Petitjean et al. $(2000)^{50}$, Pettini et al. $(2000)^{51}$, Rao \& Turnshek $(2000)^{52}$, Storrie-Lombardi \& Wolfe $(2000)^{53}$, Bowen, Tripp \& Jenkins $(2001)^{54}$, Cohen $(2001)^{55}$, Ellison et al. $(2001 \mathrm{a})^{56}$, Kanekar \& Chengalur $(2001)^{57}$, Péroux et al. $(2001)^{58}$, Turnshek et al. $(2001)^{59}$, the radio selected QSO survey of Ellison et al. (2001b) ${ }^{60}$ and finally the five new DLAs at $z>3$ occulting PSS quasars of Prochaska, Gawiser \& Wolfe $(2001)^{61}$. 


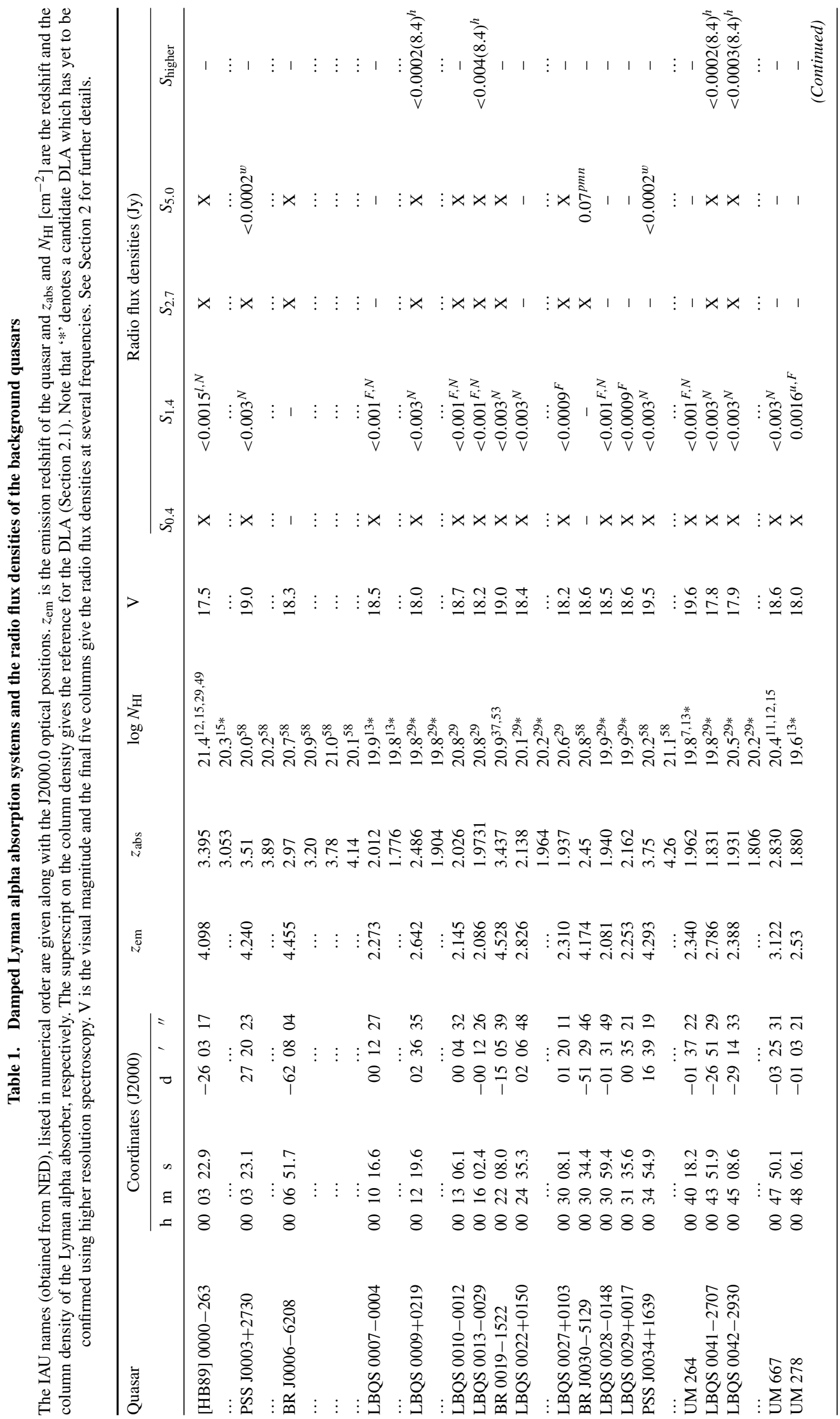




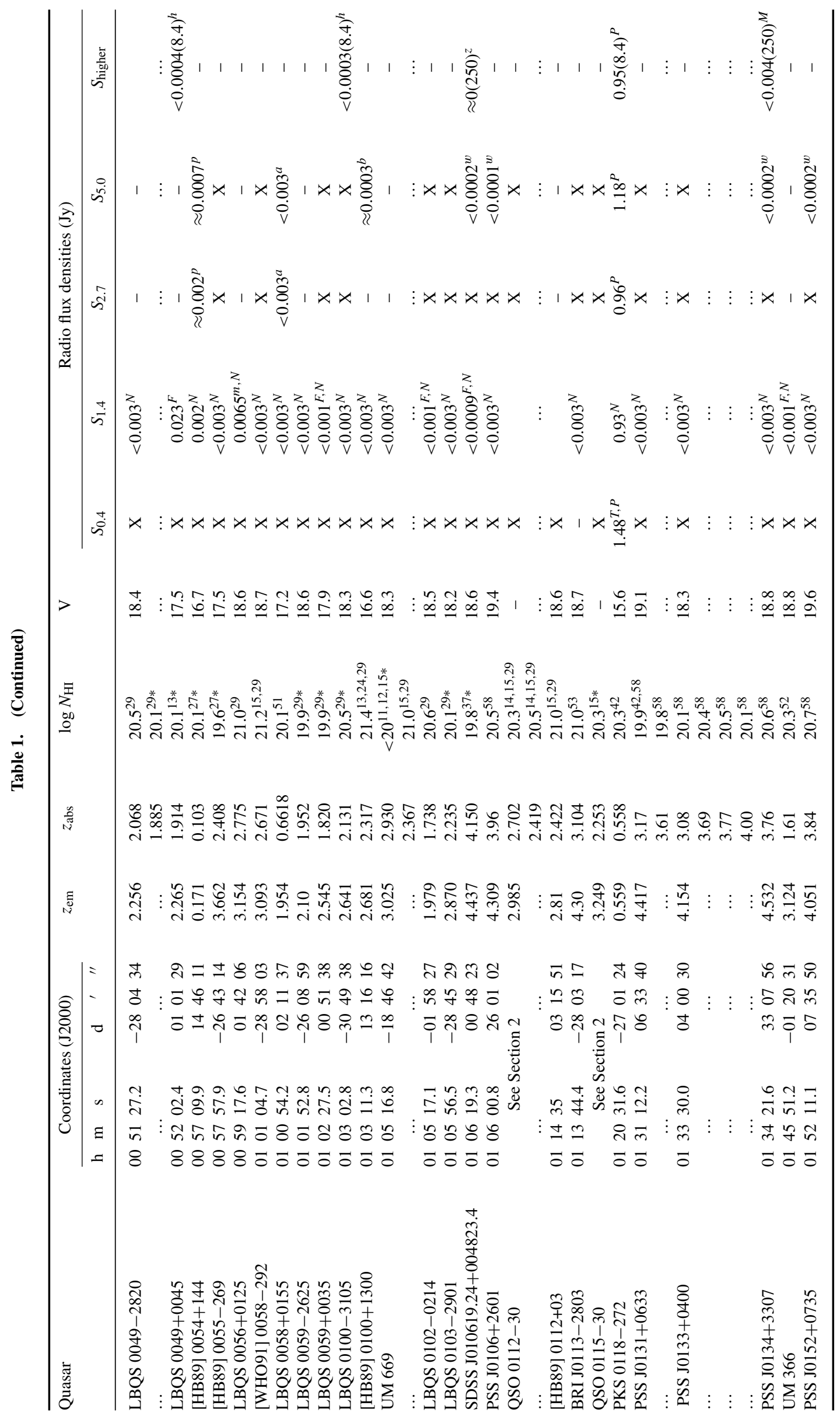




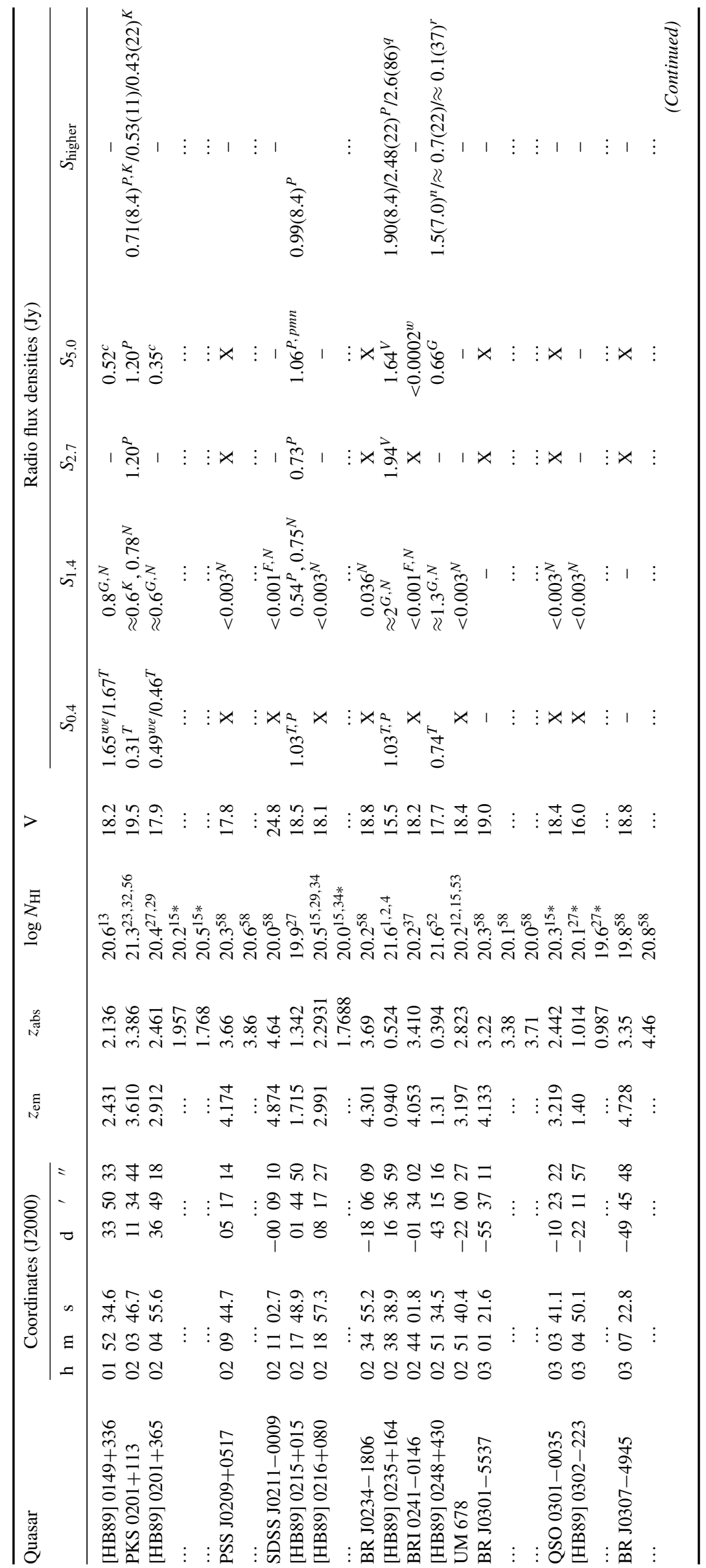




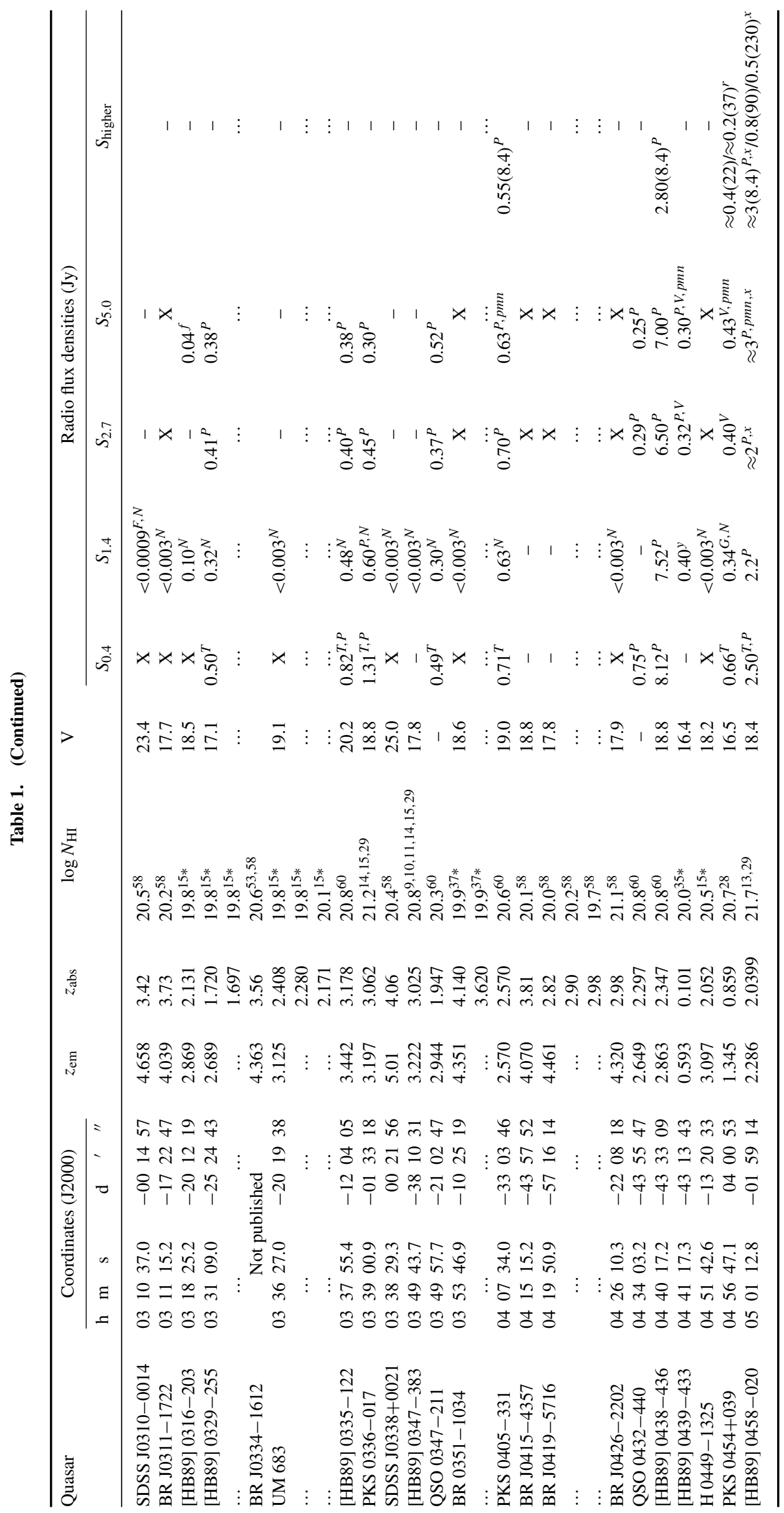




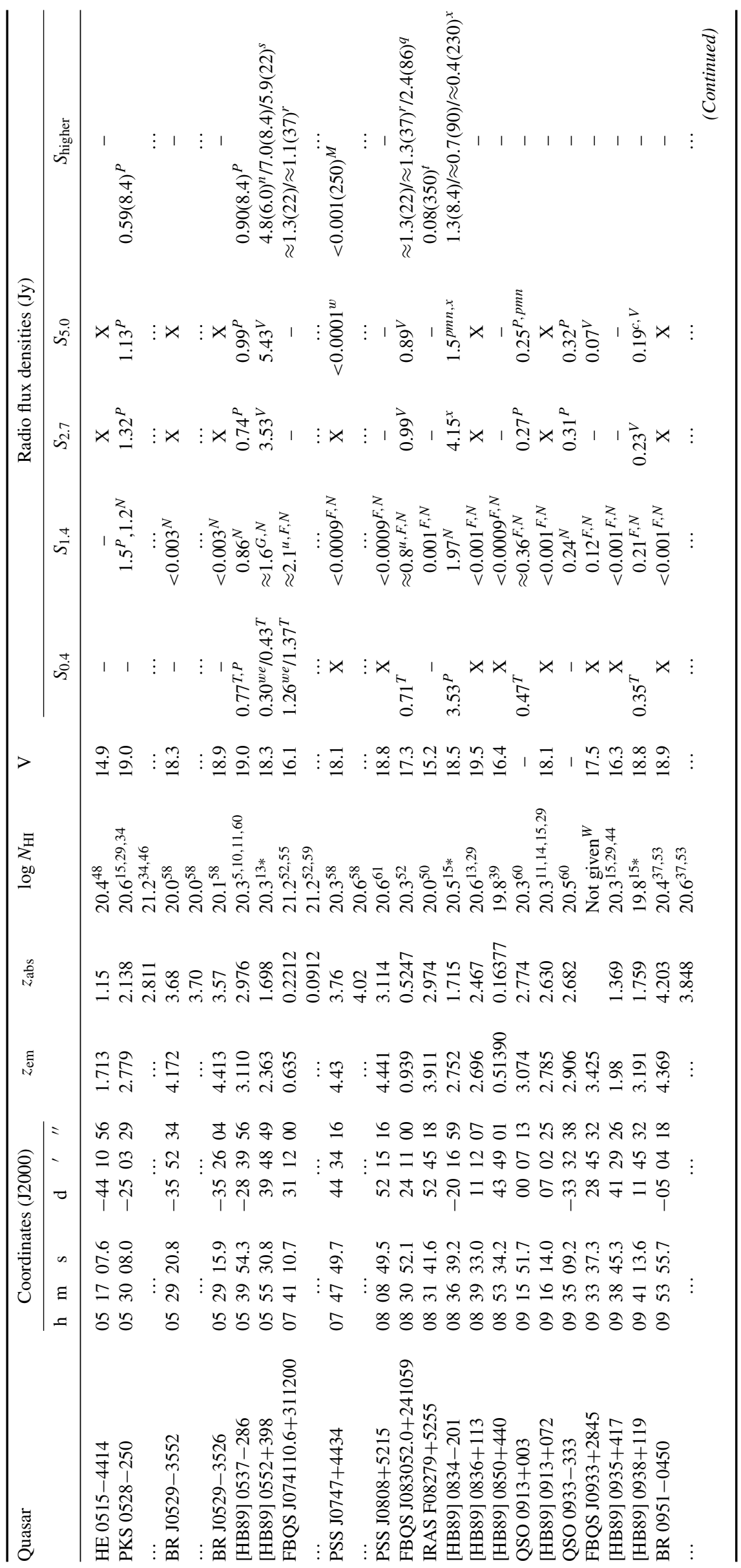




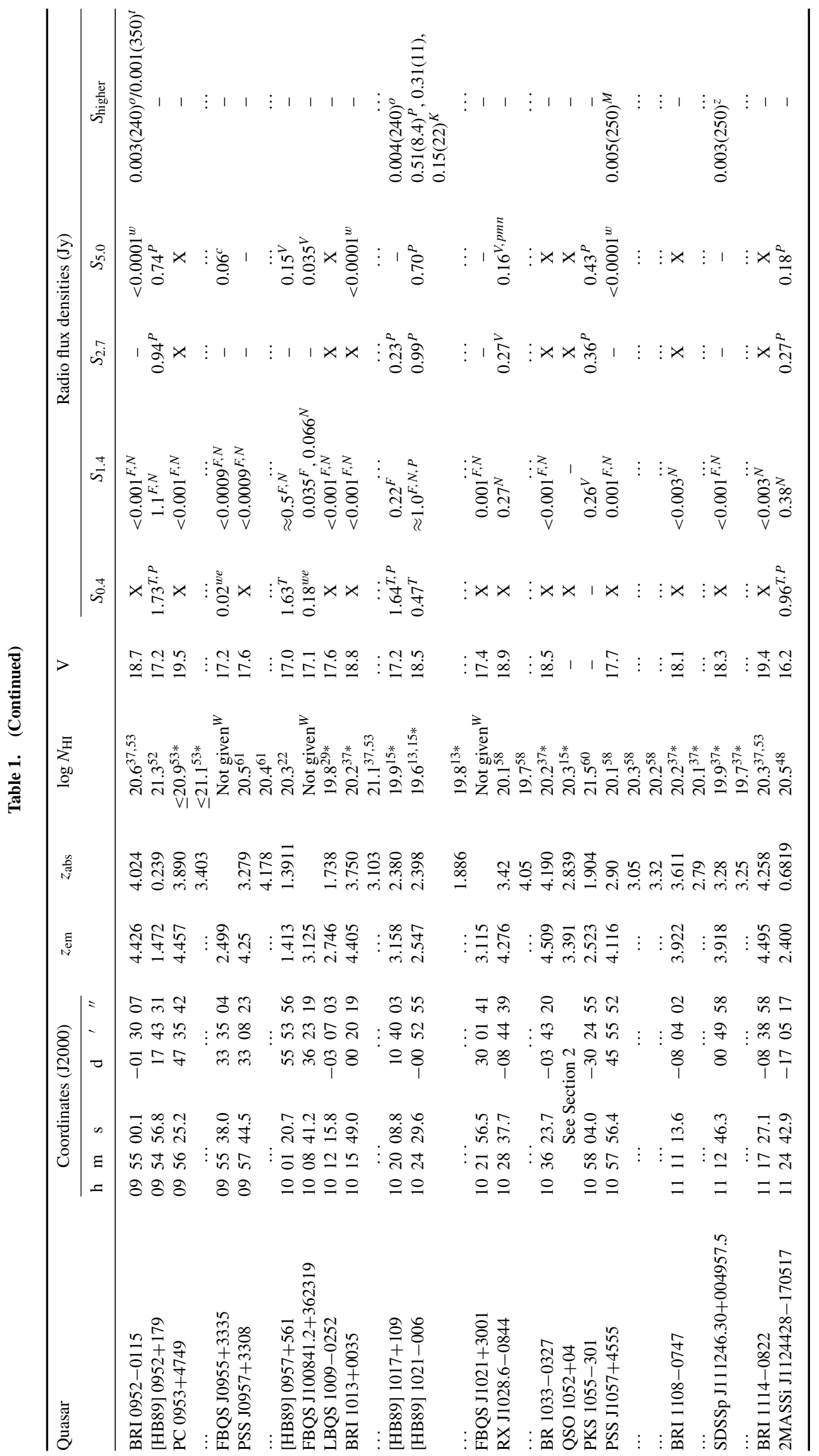




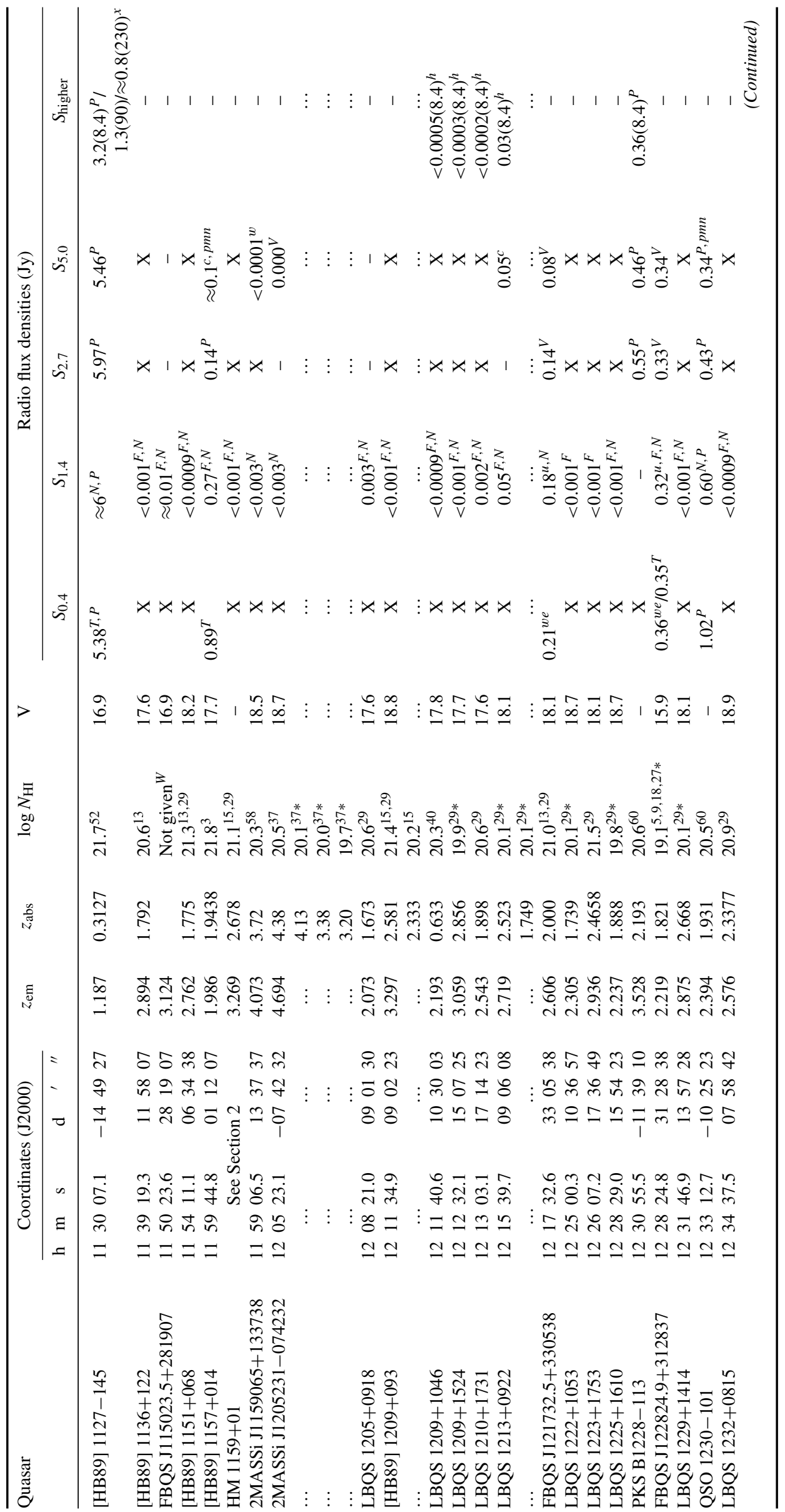




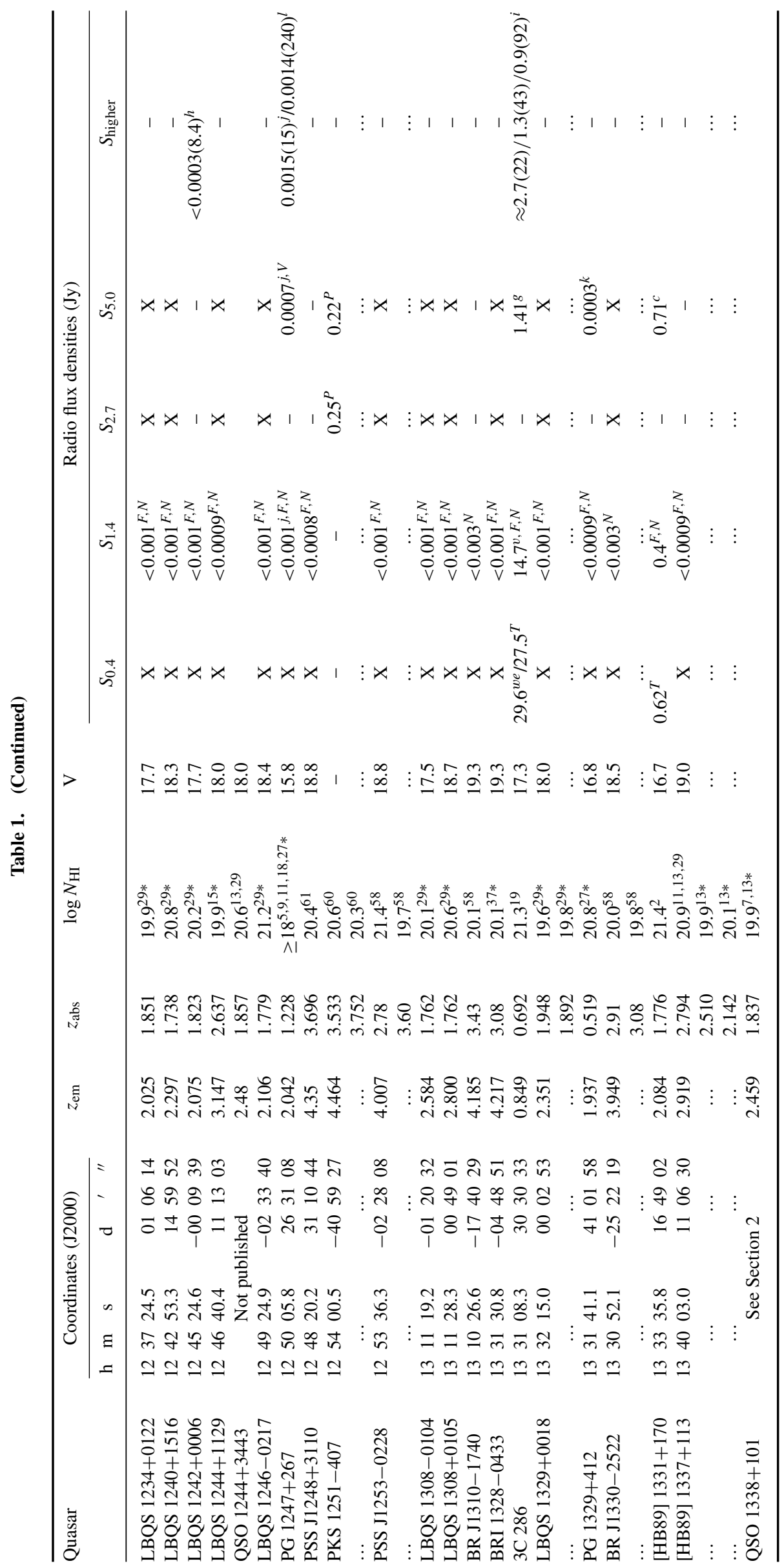




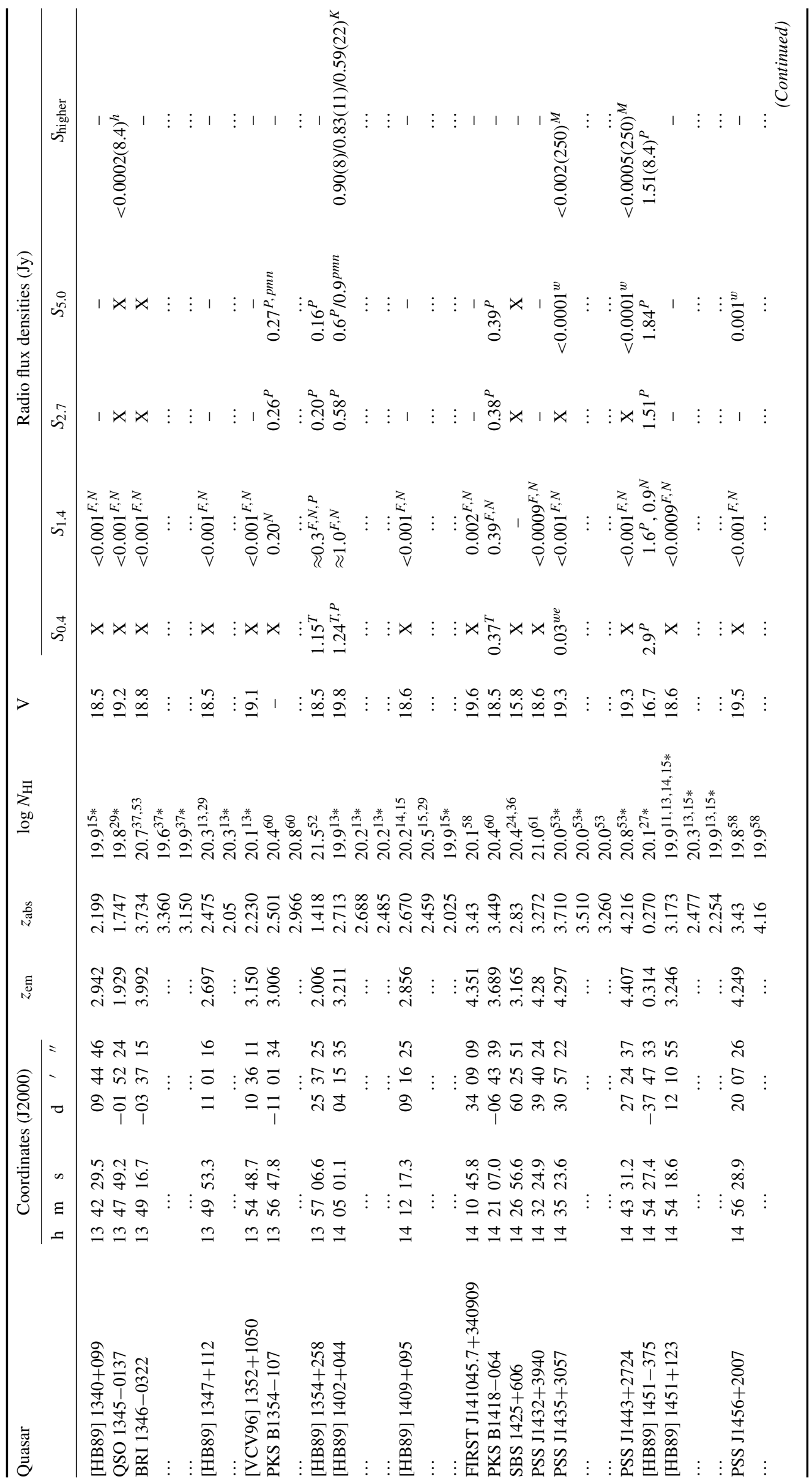




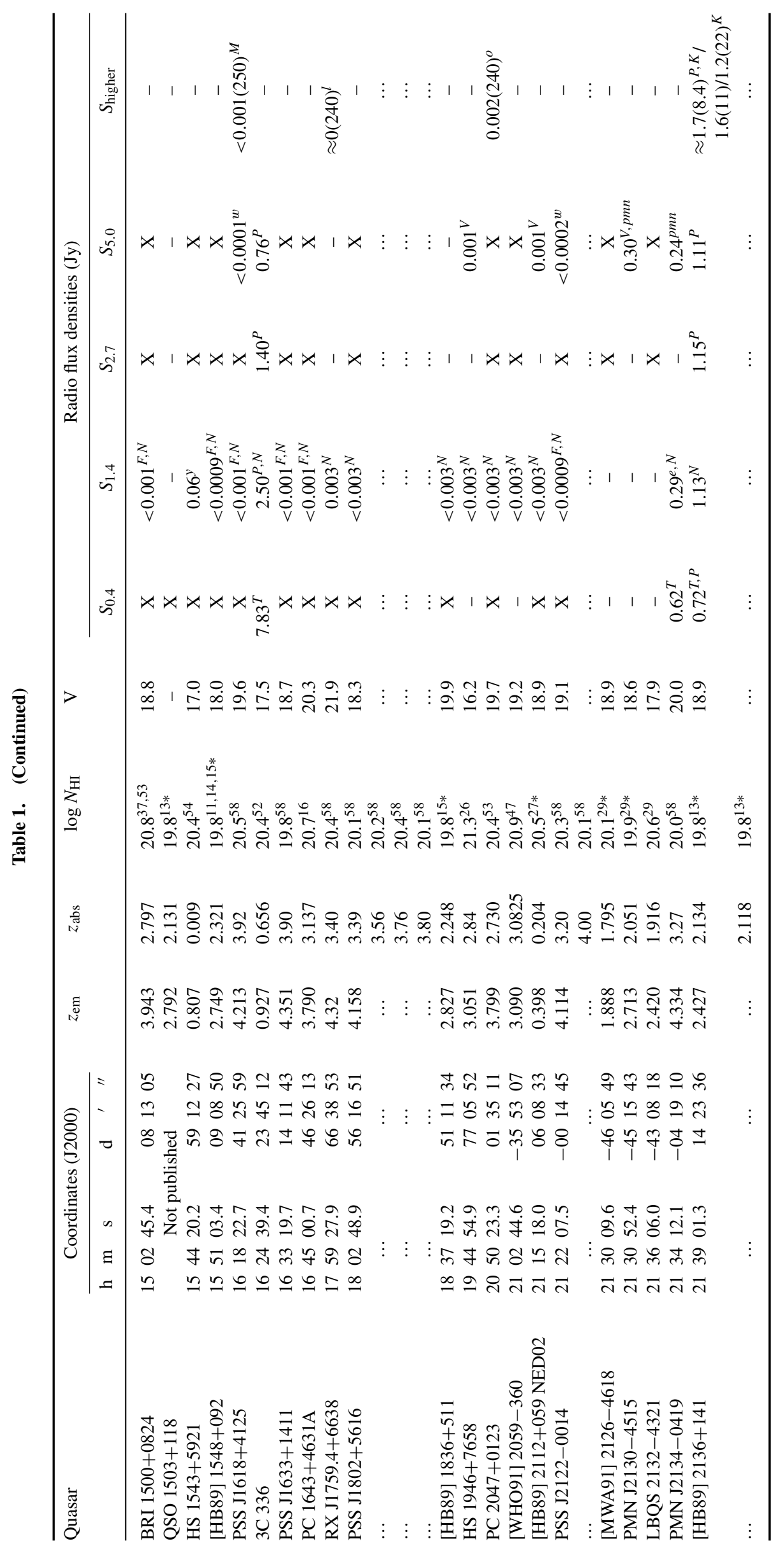




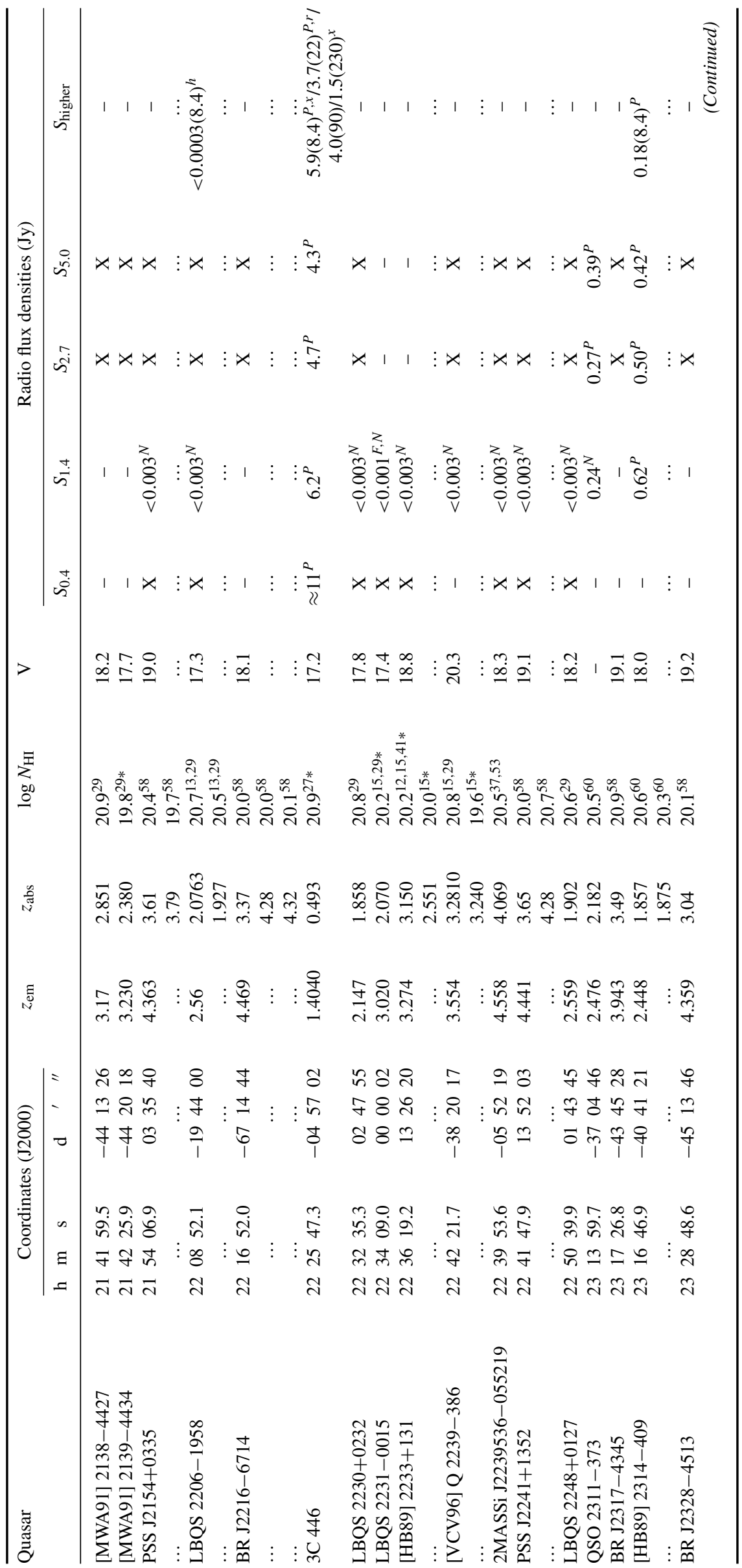




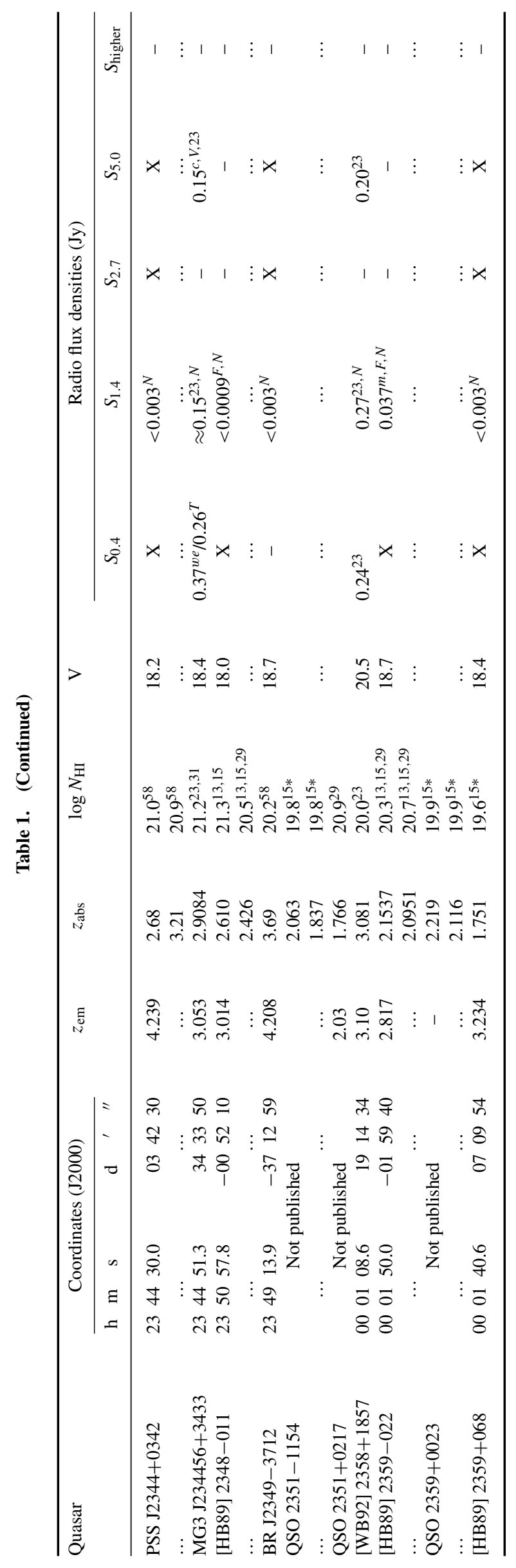


Note that several of the DLA citations do not give the background quasar's coordinates, although we did manage to get some (but not all) of these from the authors. For the remaining few we obtained the coordinates from elsewhere (e.g. NED, SIMBAD, Véron-Cetty \& Véron 2001). However, note that when checking the coordinates for the DLAs without published magnitudes against optical images (Digitized Sky and APM Surveys)*, we found no optical counterparts at the limiting APM magnitudes of 21.5 (north) and 22.5 (south) for QSOs 0112-30, 011530, 1052+04, 1159+01 (C. Hazard \& R.G. McMahon, unpublished) nor QSO 1338+101 (C. Hazard \& W.L.W. Sargent, unpublished) and so we have no knowledge of where these coordinates originally came from. Where we have failed to find optical coordinates for any of these sources, 'Not published' is inserted into the table. We have kept these sources in the catalogue for the sake of providing a full comprehensive list and, for the sake of consistency, we do not include the best radio positions as this could prove misleading.

\subsection{The Radio References}

The radio parameters of the background quasar supplying the continuum emission are compiled from Wardle \& Miley $(1971)^{a}$, Condon et al. (1981) $)^{b}$, Gregory \& Condon $(1991)^{c}$, Visnovsky et al. $(1992)^{d}$, White \& Becker $(1992)^{e}$, Lonsdale, Barthel \& Miley $(1993)^{f}$, Zhang et al. $(1994)^{g}$, Hooper et al. $(1995)^{h}$, Kameno et al. (1995) ${ }^{i}$, Barvainis, Lonsdale \& Antonucci (1996) ${ }^{j}$, Falcke, Sherwood \& Patnaik (1996) ${ }^{k}$, Omont et al. (1996) ${ }^{l}$, Bischof \& Becker (1997) ${ }^{m}$, de Vries, Barthel \& O’Dea $(1997)^{n}$, Hughes, Dunlop \& Rawlings $(1997)^{o}$, Kukula et al. (1998) $)^{p}$, Lonsdale, Doeleman \& Phillips $(1998)^{q}$, Teräsranta et al. (1998) ${ }^{r}$, Bloom et al. (1999) $)^{s}$, McMahon et al. (1999) ${ }^{t}$, Wadadekar \& Kembhavi (1999) ${ }^{u}$, Peng et al. $(2000)^{v}$, Stern et al. (2000) $)^{w}$, Tornikoski, Lainela \& Valtaoja $(2000)^{x}$, Bowen et al. (2001, and references therein) ${ }^{y}$, Carilli et al. $(2001)^{z}$ and current versions of the Parkes (Wright \& Otrupcek 1990, incorporating the $408 \mathrm{MHz}$ flux densities from the MRC catalogue; Large, Cram \& Burgess 1991) ${ }^{P}$, Green Bank (White \& Becker $1992)^{G}$, FIRST (Becker, White \& Helfand 1995) ${ }^{F}$, Texas (Douglas et al. 1996) $^{T}$, PMN (Griffith et al. 1994; Wright et al. 1994; Griffith et al. 1995; Wright et al. 1996) ${ }^{\mathrm{pmn}}$, WENSS (Rengelink et al. 1997) ${ }^{\text {we }}$, NVSS (Condon et al. $1998)^{N}$, Kovalev et al. (1999) ${ }^{K}$ and Véron-Cetty \& Véron $(2001)^{V}$ catalogues, and most recently, the MAMBO survey of the highest redshift PSS quasars (Omont et al. $2001)^{M}$. Finally, the results of White et al. $(2000)^{W}$ give new DLAs as well as the FIRST radio luminosities of the quasars illuminating these.

Note that no radio information for the quasars in Table 1 was found in the $5 \mathrm{GHz}$ catalogue of Becker, White \& Edwards (1991), the optically quiet quasar search of

*These can be found at http://archive.stsci.edu/dss/ and http://www. ast.cam.ac.uk/ apmcat/, respectively.
Kollgaard et al. (1995) nor the S5 radio source catalogue of Stickel \& Kühr (1996).

\section{Discussion}

As mentioned in the introduction, we have compiled this catalogue since the comparison between optical and radio absorption lines can provide a considerably more precise determination of $\Delta \alpha / \alpha$ : to a first approximation, the ratio of two optical transition frequencies used in the manymultiplet method (Dzuba, Flambaum \& Webb 1999; Webb et al. 1999) is $\frac{\omega_{1}}{\omega_{2}} \propto 1+0.1 \alpha^{2}$. However, the ratio of the hyperfine neutral hydrogen $(21 \mathrm{~cm})$ to an optical resonance transition frequency is directly proportional to $\alpha^{2}$, i.e. about 10 times larger. Thus, a substantial improvement in the determination of any variation of $\alpha$ could be made by obtaining further statistics from optical and $21 \mathrm{~cm}$ lines in cosmological absorbers. The limit on the variation of $\alpha$ can be obtained by the comparison of the H $21 \mathrm{~cm}$ line with any other optical or radio line (Section 3.2). However, by using redshifted $21 \mathrm{~cm} \mathrm{H}$ I together with $\alpha$-sensitive species such as iron, zinc, chromium and nickel (Dzuba et al. 1999), frequently seen in DLAs, we simultaneously maximise sensitivity and take advantage of the different signs of the frequency shifts due to $\alpha$ variation to help minimise systematic effects (Murphy et al. 2001a; Webb et al. 2001).

A new systematic effect which applies to tests for $\Delta \alpha / \alpha$ involving an $\mathrm{H} \mathrm{I}$ and optical comparison involves the possible different spatial characteristics of the radio and optical quasar emission. Large differences can result in the radio and optical light probing slightly different lines-of-sight. However, we note that there are examples where the radio and optical emission is known to coincide spatially, and those cases are clearly of particular interest (Section 3.1). In order to minimise the spatial segregation problem, the most reliable tests will come from comparing $\mathrm{H}$ I lines with neutral atomic or molecular species, or singly ionised species where the ionisation potential is smaller than that for neutral hydrogen.

\subsection{Radio-loud Quasars illuminating DLAs}

Of the known radio-loud ( $S$ radio $\gtrsim 0.1 \mathrm{Jy}$ ) systems, we summarise the current state of searches for atomic and molecular hydrogen (Section 3.2) absorption features. Note that with regard to the spatial distribution of the optical and radio emission, from the NVSS catalogue (Condon et al. 1998), unless otherwise stated, the $1.4 \mathrm{GHz}$ emission extends to a radius of $\approx 1^{\prime}$ and the peak emission coincides with the given optical position (Table 1).

PKS 0118-272: A BL Lac object where Kanekar \& Chengalur (2001) failed to detect $\mathrm{H}$ I absorption at $z=$ 0.5579 .

[HB89] 0149+336: A gravitational lens candidate for which we could find no reference to radio absorption features.

PKS 0201+113: A gravitational lens where de Bruyn et al. (1996) and Briggs, Brinks \& Wolfe (1997) have detected $\mathrm{H}$ I absorption at $z=3.388$. 
[HB89] 0201+365: No reference to radio absorption features found.

[HB89] 0215+015: A BL Lac object where Briggs \& Wolfe (1983) failed to detect H I absorption.

[HB89] 0235+164: A BL Lac object where H I absorption at $z=0.524$ has been detected (Wolfe, Briggs \& Davis 1982; Briggs \& Wolfe 1983). Douglas et al. (1992); Wiklind \& Combes (1995) failed to detect CO at the absorption redshift.

[HB89] 0248+430: Lane \& Briggs (2001) have detected the $\mathrm{H}$ I absorption at the DLA redshift.

[HB89] 0329-255: No reference to radio absorption features found.

[HB89] 0335-122: No $\mathrm{H}$ I absorption detected (N. Kanekar \& J.N. Chengalur, in preparation).

PKS 0336-017: N. Kanekar \& J.N. Chengalur (in preparation) failed to detect $\mathrm{H}$ I absorption at $z=3.0619$.

QSO 0347-211: No reference to radio absorption features found.

PKS 0405-331: As above.

QSO 0432-440: As above. No NVSS data available.

[HB89] 0438-436: Drinkwater, Combes \& Wiklind (1996) failed to detect CO in the torus of this AGN $(z=2.852)$. No NVSS data available.

[HB89] 0439-4319: Tentative H I absorption detected by Kanekar et al. (2001) in this low redshift source. No NVSS data available.

PKS 0454+039: No $\mathrm{H}_{\mathrm{I}}$ (Briggs \& Wolfe 1983) or $\mathrm{H}_{2}$ (Ge \& Bechtold 1999) absorption has been detected. No optical/radio offset, but there is a second $30^{\prime \prime}$ radius radio source centered at $5 \mathrm{~s}$ to the west.

[HB89] 0458-020: In this blazar, Wolfe et al. (1985); Briggs et al. (1989) have detected Hi absorption at $z=2.03945$. No $\mathrm{H}_{2}$ or $\mathrm{CO}$ (i.e. molecular) absorption has been detected (Wiklind \& Combes 1994b, Ge \& Bechtold 1999).

PKS 0528-250: Carilli et al. (1996) failed to detect $\mathrm{H}_{\mathrm{I}}$ absorption at $z=2.8110$, although $\mathrm{H}_{2}$ absorption in this DLA (Foltz, Chaffee \& Black 1988, Srianand \& Petitjean 1998, Ge \& Bechtold 1999) and CO emission in the $z=2.14$ DLA (Brown \& vanden Bout 1993) have been detected. Note that no $\mathrm{H}_{2}$ or $\mathrm{CO}$ absorption in either DLA was detected by Wiklind \& Combes (1994b); Lu Sargent \& Barlow (1999).

[HB89] 0537-286: No reference to radio absorption features found.

[HB89] 0552+398: Although Galactic H I (Dickey et al. 1983) and $\mathrm{HCO}^{+}$(Lucas \& Liszt 1996) absorption has been observed towards this quasar, no reference to absorption at the DLA (or any cosmological) redshift could be found.

J074110.6+311200: In this optically variable quasar, Lane et al. (1998); Kanekar, Ghosh \& Chengalur (2001) have detected $\mathrm{H}$ i absorption at $z=0.2212$.

FBQS J083052.0+241059: In this blazar, Kanekar \& Chengalur (2001) have detected $\mathrm{HI}$ absorption at $z=0.5247$.
IRAS F08279+5255: A gravitational lens in which Combes, Maoli \& Omont (1999) have detected CO $4 \rightarrow 3$ emission at $z=3.911$, the redshift of the source. There is a weak central radio source at optical position with two stronger diagonally opposing sources near $08 \mathrm{~h} 31 \mathrm{~m} 50 \mathrm{~s} / 52 \mathrm{~d} 43^{\prime} 30^{\prime \prime}$ and $08 \mathrm{~h} 31 \mathrm{~m} 25 \mathrm{~s} / 52 \mathrm{~d} 46^{\prime} 30^{\prime \prime}$.

[HB89] 0834-201: No reference to radio absorption features found for this blazar.

QSO 0913+003: No reference to radio absorption features found.

QSO 0933-333: As above. Offset from optical position at $09 \mathrm{~h} 35 \mathrm{~m} 08.6 \mathrm{~s} /-33 \mathrm{~d} 32^{\prime} 34^{\prime \prime}$.

[HB89] 0938+119: No reference to radio absorption features found. No offset but there is a second source to the south east near $09 \mathrm{~h} 41 \mathrm{~m} 20.5 \mathrm{~s} / 11 \mathrm{~d} 45^{\prime \prime} 00^{\prime}$.

[HB89] 0952+179: Kanekar \& Chengalur (2001) have detected $\mathrm{H}$ I absorption at $z=0.2378$.

[HB89] 0957+561: A gravitational lens where no H I absorption has been detected (N. Kanekar \& J.N. Chengalur, in preparation).

[HB89] 1017+109: No reference to radio absorption features found. Radio position offset $\approx 20^{\prime \prime}$ to the west of the optical centre.

[HB89] 1021-006: No reference to radio absorption features found for this optically variable quasar.

RX J1028.6-0844: No $\mathrm{H}_{\mathrm{I}}$ absorption detected (N. Kanekar \& J.N. Chengalur, in preparation).

PKS 1055-301: No reference to radio absorption features found. Radio position offset $\approx 1^{\prime}$ to the west of the optical centre.

2MASSi J1124428-170517: No reference to radio absorption features found. Offset slightly from optical position at $11 \mathrm{~h} 24 \mathrm{~m} 41.5 \mathrm{~s} /-17 \mathrm{~d} 05^{\prime} 10^{\prime \prime}$.

[HB89] 1127-145: Lane et al. (1998); Chengalur \& Kanekar (2000) have detected variable H I absorption at $z=0.3127$ towards this blazar.

[HB89] 1157+014: Wolfe et al. (1981) and Briggs \& Wolfe (1983) have detected H I absorption at $z=1.94362$. LBQS 1213+0922: No reference to radio absorption features found.

FBQS J121732.5+330538: Wiklind \& Combes (1994b) failed to detect $\mathrm{CO}$ absorption at $z=1.9984$.

FBQS J122824.9+312837: Briggs \& Wolfe (1983) failed to detect H I absorption at $z=1.7945$.

PKS B1228-113: No reference to radio absorption features found.

QSO 1230-101: As above.

PKS 1251-407: As above. No NVSS data available.

3C 286: H I at $z=0.69215$ by Brown \& Roberts (1973) but no $\mathrm{H}_{2}$ absorption has yet been detected ( $\mathrm{Ge} \&$ Bechtold 1999).

[HB89] 1331+170: A blazar where Wolfe \& Davis (1979); Briggs \& Wolfe (1983) have detected H I absorption at $z=1.7764$, but Lu et al. (1999) failed to detect CO absorption.

PKS B1354-107: No $\mathrm{H}_{\mathrm{I}}$ absorption detected (N. Kanekar \& J.N. Chengalur, in preparation) in the 
Table 2. The radio-loud DLAs in which $\mathrm{H}_{\mathrm{I}}$ absorption has been searched

$\tau$ is the optical depth of the $\mathrm{H}_{\mathrm{I}}$ line, with $3 \sigma$ upper limits quoted, as given by the references in Section 3.1 . For PKS $0201+113$ the values are from de Bruyn et al. (1996) and Briggs et al. (1997), respectively. PKS 0336-017 and MG3 J234456+3433 - these are results from N. Kanekar \& J.N. Chengalur (in preparation); Carilli et al. (1996) obtained $\tau<0.02$ and 0.1 , respectively. S is the approximate flux density in janskys at $z_{\mathrm{abs}}$ and S.I. is the spectral index (both are estimated from the flux density values in Table 1 and $\mathrm{S} \propto \nu^{-S . I}$ ). In the last column, GPS designates a GHz peaked source with the approximate turnover frequency given in parenthesis. In the case of the 'U-shaped' SEDs, [HB89] 0215+015 is known to exhibit radio outbursts (e.g. Ledden \& Odell 1985) and so the flux densities quoted will be variable. For PKS $0454+039$ and [HB89] 1331+170, these could be due to an anomalous flux density measurement and both are considered flat spectrum sources (e.g. Wampler et al. 1984; Mattox et al. 1997).

\begin{tabular}{|c|c|c|c|c|c|}
\hline Quasar & $\tau$ & $\log N_{\mathrm{HI}}$ & $S$ & S.I. & Notes \\
\hline PKS 0118-272 & $<0.007$ & 20.3 & 1.2 & 0.1 & \\
\hline PKS $0201+113$ & $0.09,0.04$ & 21.3 & 0.3 & - & GPS (2.6) \\
\hline [HB89] 0215+015 & $<0.04$ & 19.9 & 0.9 & - & See caption \\
\hline [HB89] $0235+164$ & $0.05-0.5$ & 21.6 & 1.8 & -0.2 & Inverted \\
\hline$[\mathrm{HB} 89] 0248+430$ & 0.20 & 21.6 & 1.2 & - & GPS (2.5) \\
\hline [HB89] 0335-122 & $<0.008$ & 20.8 & 0.8 & 0.3 & \\
\hline PKS 0336-017 & $<0.005$ & 21.2 & 1.3 & 0.6 & \\
\hline [HB89] 0439-4319 & $<0.007$ & 20.0 & 0.4 & 0.2 & \\
\hline PKS 0454+039 & $<0.01$ & 20.7 & 0.4 & - & See caption \\
\hline [HB89] 0458-020 & 0.3 & 21.7 & 2.5 & 0.3 & \\
\hline PKS 0528-250 & $<0.2$ & 21.2 & 1.9 & 0.5 & For $z_{\mathrm{abs}}=2.811 \mathrm{DLA}$ \\
\hline J074110.6+311200 & 0.07 & 21.2 & 1.9 & - & GPS (2.9) $z_{\mathrm{abs}}=0.221 \mathrm{DLA}$ \\
\hline FBQS J083052.0+241059 & 0.007 & 20.3 & 0.8 & -0.2 & Inverted \\
\hline [HB89] 0952+179 & 0.013 & 21.3 & 1.2 & 0.3 & \\
\hline$[\mathrm{HB} 89] 0957+561$ & $<0.004$ & 20.3 & 0.9 & 1.3 & \\
\hline RX J1028.6-0844 & $<0.03$ & 20.1 & 1.7 & 0.9 & $z_{\mathrm{abs}}=3.42 \mathrm{DLA}$ \\
\hline [HB89] 1127-145 & 0.06 & 21.7 & 6.2 & - & GPS (1.4) \\
\hline [HB89] 1157+014 & 0.05 & 21.8 & 1.0 & 0.8 & \\
\hline FBQS J122824.9+312837 & $<0.05$ & 19.1 & 0.3 & 0 & \\
\hline $3 \mathrm{C} 286$ & 0.11 & 21.3 & 19.0 & 0.6 & \\
\hline [HB89] $1331+170$ & 0.020 & 21.4 & 0.6 & - & See caption \\
\hline PKS B1354-107 & $<0.05$ & 20.8 & 0.2 & 0 & $z_{\mathrm{abs}}=2.996 \mathrm{DLA}$ \\
\hline [HB89] 1451-375 & $<0.006$ & 20.1 & 1.8 & 0.2 & \\
\hline $3 \mathrm{C} 446$ & $<0.02$ & 20.9 & 7.4 & 0.5 & \\
\hline MG3 J234456+3433 & $<0.04$ & 21.2 & 0.3 & 0.2 & \\
\hline
\end{tabular}

$z_{\text {abs }}=2.966$ DLA. Radio position offset $\approx 15^{\prime \prime}$ to the west of the optical centre.

[HB89] 1354+258: No reference to radio absorption features found.

[HB89] 1402+044: No reference to radio absorption features found for this BL Lac.

PKS B1418-064: No reference to radio absorption features found.

[HB89] 1451-375: Chengalur \& Kanekar (2000) failed to detect $\mathrm{H}_{\mathrm{I}}$ absorption in this HST source.

3C 336: No reference to radio absorption features found for this optically variable quasar.

PMN J2130-4515: No reference to radio absorption features found. No NVSS data available.

PMN J2134-0419: No reference to radio absorption features found.

[HB89] 2136+141: No reference to radio absorption features found.

3C 446: A blazar not detected in $\mathrm{H}_{\mathrm{I}}$ absorption at the DLA (Chengalur \& Kanekar 2000) nor CO absorption at the quasar redshift (Drinkwater et al. 1996).

QSO 2311-373: No reference to radio absorption features found.
[HB89] 2314 -409: As above. No NVSS data available. MG3 J234456+3433: Carilli et al. (1996) and N. Kanekar \& J.N. Chengalur (in preparation) failed to detect $\mathrm{HI}$ absorption at $z=2.9084$.

Finally, note that $\mathrm{HI}$ absorption has been observed in the inferred (from metal lines) DLAs 3 C196, LBQS 1229-0207 (Wolfe et al. 1995) and [HB89] 1243-072 (Lane \& Briggs 2001).

\subsection{Searching for New Radio Absorbers}

If we summarise the current $\mathrm{H}$ I absorption results for the DLAs (Table 2), we see that although many of the positive results have very high column densities, this does not appear to be a prerequisite for $\mathrm{H}$ I absorption (i.e. FBQS J083052.0+241059). Perhaps also of relevance is the spectral energy distributions (SEDs): note that all of the $\mathrm{GHz}$ peaked sources have high column densities and have all been detected in H I. Of the two inverted SEDs, one DLA has a high column density whereas the other is relatively low and both of the flat SED detections have high column densities. Finally, the two steep spectrum quasars which illuminate DLAs detected in $\mathrm{H}$ I absorption 
([HB89] 1157+014 and 3C 286) also have high column densities.

Because of the relation between turnover frequency and source size (Fanti et al. 1990; O'Dea \& Baum 1997), we may expect a higher $\mathrm{H} \mathrm{I}$ absorption detection rate from flat and inverted SED sources, since these result from similar optical and radio lines-of-sight. However, as it stands, the statistics are too small (Table 2) and so in order to maximise our sample, it appears that the way to proceed is an unbiased search for $\mathrm{H}_{\mathrm{I}}$ in the DLAs occulting the remaining radio-loud quasars.

As mentioned in Section 1, as well as optical and $\mathrm{H}_{\mathrm{I}}$ comparisons, the inter-comparison of atomic and molecular lines will also give a ten-fold increase in accuracy for $\Delta \alpha / \alpha$ : due to its zero dipole moment and small moment of inertia, molecular hydrogen cannot be directly observed at radio frequencies* and so it is the usual practice to infer the presence of this from the millimetre rotational lines of such molecules as CO. In order to also take advantage of this, we have applied for time to search for molecular absorption lines in the DLAs occulting mm-loud quasars with the IRAM 30 metre and Swedish ESO Sub-millimetre telescopes. Recently (April 2002), we have been awarded time on the Australia Telescope Compact Array in order to obtain $90 \mathrm{GHz}$ flux measurements for the whole radioloud sample, as a means of selecting new sources in which to search for millimetre absorption. The results will be published in forthcoming papers.

Also, with regard to finding new systems in which there may be absorption (in all three frequency regimes), we see that 13 of the quasars are known to be BL Lac/optically variable/blazars ${ }^{\dagger}$ and that 3 of the sources are known gravitational lenses. This may be of interest as of the four known high redshift millimetre (i.e. molecular) absorbers, two are BL Lac objects: B 0218+357 (Wiklind \& Combes 1995) and PKS 1413+135 (Wiklind \& Combes 1994a) This may suggest several strategies for finding similar new absorbers (Stocke \& Rector 1999) which could prove useful in appending to this catalogue.

\section{Summary}

We have performed an exhaustive search of the literature in order to produce a list of all known damped Lyman alpha systems and their associated radio properties. It is the 57

\footnotetext{
* In the case of $z>1.8$ sources, however, the ultraviolet lines of $\mathrm{H}_{2}$ are redshifted into the optical window, making molecular hydrogen readily observable at these frequencies. As well as for PKS 0528-250 (Section 3.1) molecular hydrogen has also been detected in the DLAs occulting the radio-quiet quasars [HB89] 0000-263 (Levshakov et al. 2000), LBQS 0013-0029 (Ge \& Bechtold 1997; Petitjean, Srianand, \& Ledoux 2002), [HB89] 0347-383 (Levshakov et al. 2002), LBQS 1232+0815 (Ge \& Bechtold 1997; Srianand et al. 2000) and the inferred (Wolfe et al. 1995) DLA [HB89] 0551-366 (Ledoux, Srianand, \& Petitjean 2002).

${ }^{\dagger}$ BL Lacs and Optically Violent Variables are known collectively as blazars. In these radio-loud active galactic nuclei the radio jet is relativistically beamed close to the line-of-sight (e.g. Peterson 1997).

¥The former, as well as PKS 1830-211, is also a gravitational lens (Wiklind \& Combes 1996).
}

radio-loud systems in which we are interested as many of these have the potential to show $\mathrm{H}$ I absorption in each DLA. Of the sources searched, it is seen that several exhibit such absorption and we are involved in an ongoing project to search for this in the remaining systems. Not only will this give us radio lines for comparison with optical data in order to constrain any temporal variations in the fine structure constant, but we will have a significant sample from which we could consider why some DLAs absorb in H I whereas others do not. For example, Chengalur \& Kanekar (2000) suggest that low redshift DLAs may arise from a multitude of absorbers, and hence do not have sufficient path length for $\mathrm{H}$ I absorption, while those of higher redshift are due to more compact systems. Finally, as well as finding new $\mathrm{H}$ I absorbers, we hope that this catalogue will prove useful to those using damped Lyman alpha systems as part of their research.

Final note: In order to retain its usefulness to the astronomical community, we have now produced an on-line version of this catalogue which will be continually updated. This is available from http://www.phys.unsw. edu.au/ $\sim$ sjc/dla.

\section{Acknowledgments}

We wish to thank the John Templeton Foundation for supporting this work, the two anonymous referees and Barry Madore at NED for their comments, Sandra Ricketts of the Anglo-Australian Observatory library as well as Fredrik Rantakyrö, Sara Ellison, Ken Lanzetta and especially Nissim Kanekar for giving us the preliminary results of his Hi survey. This research has made use of the NASA/IPAC Extragalactic Database (NED) ${ }^{\S}$ as well as the VizieR database of CDS catalogues (Ochsenbein, Bauer \& Marcout 2000) $)^{\mathrm{I}}$.

\section{References}

Bahcall, J. N., Joss, P. C., \& Lynds, R. 1973, ApJ, 182, L95

Bahcall, J. N., et al. 1993, ApJS, 87, 1

Bahcall, J. N., et al. 1996, ApJ, 457, 19

Barvainis, R., Lonsdale, C., \& Antonucci, R. 1996, AJ, 111, 1431

Beaver, E. A., Cohen, R. D., \& Junkkarinen, V. T. 1992, in American Astronomical Society Meeting, 4803

Bechtold, J., Green, R. F., Weymann, R. J., Schmidt, M., Estabrook, F. B., Sherman, R. D., Wahlquist, H. D., \& Heckman, T. M. 1984, ApJ, 281, 76

Becker, R. H., White, R. L., \& Edwards, A. L. 1991, ApJS, 75, 1 Becker, R. H., White, R. L., \& Helfand, D. J. 1995, ApJ, 450, 559 Bertoldi, F., et al. 2000, A\&A, 360, 92

Bischof, O. B., \& Becker, R. H. 1997, AJ, 113, 2000

Black, J. H., Chaffee, F. H., \& Foltz, C. B. 1987, ApJ, 317, 442

Bloom, S. D., Marscher, A. P., Moore, E. M., Gear, W., Teräsranta, H., Valtaoja, E., Aller, H. D., \& Aller, M. F. 1999, ApJS, 122, 1

Bowen, D. V., Tripp, T. M., \& Jenkins, E. B. 2001, AJ, 121, 1456

$\S$ Operated by the Jet Propulsion Laboratory, California Institute of Technology, under contract with the National Aeronautics and Space Administration.

Ihttp://vizir.u-strasbg.fr/local/cgi-bin/vizHelp?faq.htx 
Bowen, D. V., Huchtmeier, W., Brinks, E., Tripp, T. M., \& Jenkins, E. B. 2001, A\&A, 372, 820

Briggs, F. H., \& Wolfe, A. M. 1983, ApJ, 268, 76

Briggs, F. H., Brinks, E., \& Wolfe, A. M. 1997, AJ, 113, 467

Briggs, F. H., Wolfe, A. M., Liszt, H. S., Davis, M. M., \& Turner, K. L. 1989, ApJ, 341, 650

Brown, R. L., \& Roberts, M. S. 1973, ApJ, 184, L7

Brown, R. L., \& vanden Bout, P. A. 1993, ApJ, 412, L21

Carilli, C. L., Lane, W., de Bruyn, A. G., Braun, R., \& Miley, G. K. 1996, AJ, 111, 1830

Carilli, C. L., et al. 2000, ApJ, 533, L13

Carilli, C. L., et al. 2001, ApJ, 555, 625

Chaffee, F. H., Stepanian, J. A., Chavushian, V. A., Foltz, C. B., \& Green, R. F. 1994, in American Astronomical Society Meeting, 1805

Chengalur, J. N., \& Kanekar, N. 2000, MNRAS, 318, 303

Cohen, J. G. 2001, AJ, 121, 1275

Combes, F., \& Wiklind, T. 1999, in Highly Redshifted Radio Lines, ASP Conf. Series 156, ed. C. L. Carilli, S. J. E. Radford, K. M. Menten, \& G. I. Langston (San Frcncisco: ASP), 210

Combes, F., Maoli, R., \& Omont, A. 1999, A\&A, 345, 369

Condon, J. J., Odell, S. L., Puschell, J. J., \& Stein, W. A. 1981, ApJ, 246,624

Condon, J. J., Cotton, W. D., Greisen, E. W., Yin, Q. F., Perley, R. A., Taylor, G. B., \& Broderick, J. J. 1998, AJ, 115, 1693

Corbelli, E., Salpeter, E. E., \& Bandiera, R. 2001, ApJ, 550, 26

Courvoisier, T.-L., \& Paltani, S. 1992, IUE-ULDA Access Guide No. 4A, ESA, SP 1153

Cowie, L. L., \& Songaila, A., 1995, ApJ, 453, 596

de Bruyn, A. G., O'Dea, C. P., \& Baum, S. A. 1996, A\&A, 305, 450

de la Varga, A., Reimers, D., Tytler, D., Barlow, T., \& Burles, S. 2000, A\&A, 363, 69

de Vries, W. H., Barthel, P. D., \& O’Dea, C. P. 1997, A\&A, 321, 105

Dickey, J. M., Kulkarni, S. R., Heiles, C. E., \& van Gorkom, J. H. 1983, ApJS, 53, 591

D’Odorico, S., Dessauges-Zavadsky, M., \& Molaro, P. 2001, A\&A, $368, \mathrm{~L} 21$

Douglas, N. G., Radford, S. J. E., Roland, J., \& Webb, J. K. 1992, A\&A, 262, 8

Douglas, J. N., Bash, F. N., Bozyan, F. A., Torrence, G. W., \& Wolfe, C. 1996, AJ, 111, 1945

Drinkwater, M. J., Combes, F., \& Wiklind, T. 1996, A\&A, 312, 771

Drinkwater, M. J., Webb, J. K., Barrow, J. D., \& Flambaum, V. V. 1998, MNRAS, 295, 457

Dzuba, V. A., Flambaum, V. V., \& Webb, J. K. 1999, PhRvA, 59, 230

Ellison, S. L., Pettini, M., Steidel, C. C., \& Shapley, A. E. 2001a, ApJ, 549, 770

Ellison, S. L., Yan, L., Hook, I. M., Pettini, M., Wall, J. V., \& Shaver, P. 2001b, A\&A, 379, 393

Falcke, H., Sherwood, W., \& Patnaik, A. R. 1996, ApJ, 471, 106

Fanti, R., Fanti, C., Schilizzi, R. T., Spencer, R. E., Nan Rendong, Parma, P., van Breugel, W. J. M., \& Venturi, T. 1990, A\&A, 231, 333

Foltz, C. B., Chaffee, F. H., \& Black, J. H. 1988, ApJ, 324, 267

Ge, J., \& Bechtold, J. 1997, ApJ, 477, L73

Ge, J., \& Bechtold, J. 1999, in Highly Redshifted Radio Lines, ASP Conference Series, ed. C. Carilli, S. Radford, K. Menton, \& G. Langston (San Francisco: ASP), 121

Gerin, M., Phillips, T. G., Benford, D. J., Young, K. H., Menten, K. M., \& Frye, B. 1997, ApJ, 488, L31

Gregory, P. C., \& Condon, J. J. 1991, ApJS, 75, 1011

Griffith, M. R., Wright, A. E., Burke, B. F., \& Ekers, R. D. 1994, ApJS, 90, 179

Griffith, M. R., Wright, A. E., Burke, B. F., \& Ekers, R. D. 1995, ApJS, 97, 347

Hooper, E. J., Impey, C. D., Foltz, C. B., \& Hewett, P. C. 1995, ApJ, 445,62
Hughes, D. H., Dunlop, J. S., \& Rawlings, S. 1997, MNRAS, 289, 766

Impey, C. D., Petry, C. E., Malkan, M. A., \& Webb, W. 1996, ApJ, 463,473

Ivison, R. J., Harrison, A. P., \& Coulson, I. M. 1998, A\&A, 330, 443

Jannuzi, B. T., et al. 1998, ApJS, 118, 1

Jimenez, R., Bowen, D. V., \& Matteucci, F. 1999, ApJ, 514, L83

Kameno, S., et al. 1995, PASJ, 47, 711

Kanekar, N., \& Chengalur, J. N. 2001, A\&A, 369, 42

Kanekar, N., Ghosh, T., \& Chengalur, J. N. 2001, A\&A, 373, 394

Kanekar, N., Chengalur, J. N., Subrahmanyan, R., \& Petitjean, P. 2001, A\&A, 367, 46

Klein, U., Vigotti, M., Gregorini, L., Reuter, H.-P., Mack, K.-H., \& Fanti, R. 1996, A\&A, 313, 417

Kollgaard, R. I., Feigelson, E. D., Laurent-Muehleisen, S. A., Spinrad, H., Dey, A., \& Brinkmann, W. 1995, ApJ, 449, 61

Kovalev, Y. Y., Nizhelsky, N. A., Kovalev, Y. A., Berlin, A. B., Zhekanis, G. V., Mingaliev, M. G., \& Bogdantsov, A. V. 1999, A\&AS, 139, 545

Kukula, M. J., Dunlop, J. S., Hughes, D. H., \& Rawlings, S. 1998, MNRAS, 297, 366

Lane, W. M., \& Briggs, F. H. 2001, ApJ, 561, L27

Lane, W., Smette, A., Briggs, F. H., Rao, S. M., Turnshek, D. A., \& Meylan, G. 1998, AJ, 116, 26

Lanzetta, K. M. 1988, ApJ, 332, 96

Lanzetta, K. M. 1991, ApJ, 375, 1

Lanzetta, K. M., Wolfe, A. M., \& Turnshek, D. A. 1995, ApJ, 440,435

Lanzetta, K. M., Wolfe, A. M., Turnshek, D. A., Lu, L., McMahon, R. G., \& Hazard, C. 1991, ApJS, 77, 1

Lanzetta, K. M., Yahata, N., Pascarelle, S., Chen, H.-W., \& Fernándes-Soto, A. 2002, ApJ, submitted

Lanzetta, K. M., et al. 1997, AJ, 114, 1337

Large, M. I., Cram, L. E., \& Burgess, A. M. 1991, The Observatory, 111,72

Le Brun, V., Viton, M., \& Milliard, B. 1998, A\&A, 340, 381

Le Brun, V., Bergeron, J., Boisseé, P., \& Deharveng, J. M. 1997, A\&A, 321, 733

Ledden, J. E., \& Odell, S. L. 1985, ApJ, 298, 630

Ledoux, C., Srianand, R., \& Petitjean, P. 2002, A\&A, accepted (astro-ph/0206463)

Leibundgut, B., \& Robertson, J. G. 1999, MNRAS, 303, 711

Levshakov, S. A., Molaro, P., Centurión, M., D’Odorico, S., Bonifacio, P., \& Vladilo, G. 2000, A\&A, 361, 803

Levshakov, S. A., Dessauges-Zavadsky, M., D’Odorico, S., \& Molaro, P. 2002, ApJ, 565, 696

Lonsdale, C. J., Barthel, P. D., \& Miley, G. K. 1993, ApJS, 87, 63

Lonsdale, C. J., Doeleman, S. S., \& Phillips, R. B. 1998, AJ, 116, 8

Lu, L., Sargent, W. L. W., \& Barlow, T. A. 1997, ApJ, 484, 131

Lu, L., Sargent, W. L. W., \& Barlow, T. A. 1999, in Highly Redshifted Radio Lines, ASP Conf. Series 156, ed. C. L. Carilli, S. J.E. Radford, K. M. Menten, \& G. I. Langston (San Francisco: ASP), 132

Lu, L., Sargent, W. L. W., Barlow, T. A., Churchill, C. W., \& Vogt, S. S. 1996, ApJS, 107, 475

Lu, L., Savage, B. D., Tripp, T. M., \& Meyer, D. M. 1995, ApJ, 447,597

Lu, T. L., Wolfe, A. M., Turnshek, D. A., \& Lanzetta, K. M. 1993, ApJS, 84, 1

Lucas, R., \& Liszt, H. 1996, A\&A, 307, 237

Matteucci, F., Molaro, P., \& Vladilo, G. 1997, A\&A, 321, 45

Mattox, J. R., Schachter, J., Molnar, L., Hartman, R. C., \& Patnaik, A. R. 1997, ApJ, 481, 95

McMahon, R. G., Priddey, R. S., Omont, A., Snellen, I., \& Withington, S. 1999, MNRAS, 309, L1

Meyer, D. M., \& York, D. G. 1992, ApJ, 399, L121

Meyer, D. M., Black, J. H., Chaffee, F. H., Foltz, C. B., \& York, D. G. 1986, ApJ, 308, L37 
Molaro, P., Bonifacio, P., Centurión, M., D’Odorico, S., Vladilo, G. Santin, P., \& Di Marcantonio, P. 2000, ApJ, 541, 54

Murphy, M. T., Webb, J. K., Flambaum, V. V., Churchill, C. W., \& Prochaska, J. X. 2001a, MNRAS, 327, 1223

Murphy, M. T., Webb, J. K., Flambaum, V. V., Drinkwater, M. J., Combes, F., \& Wiklind, T., 2001b, MNRAS, 327, 1244

Ochsenbein, F., Bauer, P., \& Marcout, J. 2000, A\&AS, 143, 23

O'Dea, C. P., \& Baum, S. A. 1997, AJ, 113, 148

Omont, A., Cox, P., Bertoldi, F., McMahon, R. G., Carilli, C., \& Isaak, K. G. 2001, A\&A, 374, 371

Omont, A., McMahon, R. G., Cox, P., Kreysa, E., Bergeron, J., Pajot, F., \& Storrie-Lombardi, L. J. 1996, A\&A, 315, 1

Pei, Y. C., Fall, S. M., \& Hauser, M. G. 1999, ApJ, 522, 604

Peng, B., Kraus, A., Krichbaum, T. P., \& Witzel, A. 2000, A\&AS, 145,1

Péroux, C., McMahon, R. G., Storrie-Lombardi, L. J., Irwin, M. 2002, MNRAS, submitted (astro-ph/0107045)

Péroux, C., Storrie-Lombardi, L. J., McMahon, R. G., Irwin, M., Hook, I. M. 2001, AJ, 121, 1799

Peterson, B. M. 1997, An Introduction to Active Galactic Nuclei (Cambridge: Cambridge University Press)

Petitjean, P., Srianand, R., \& Ledoux, C. 2002, MNRAS, 332, 383

Petitjean, P., Aracil, B., Srianand, R., \& Ibata, R. 2000, A\&A, 359,457

Petitjean, P., Théodore, B., Smette, A., Lespine, Y. 1996, A\&A, 313, L25

Pettini, M., \& Bowen, D. V., 2001, ApJ, 560, 41

Pettini, M., Boksenberg, A., \& Hunstead, R. W. 1990, ApJ, 348, 48

Pettini, M., King, D. L., Smith, L. J., \& Hunstead, R. W. 1997, ApJ, 478, 536

Pettini, M., Ellison, S. L., Steidel, C. C., Shapley, A. E., \& Bowen, D. V. 2000, ApJ, 532, 65

Prochaska, J. X., Gawiser, E., \& Wolfe, A. M. 2001, ApJ, 552, 99

Rao, S. M., \& Turnshek, D. A. 2000, ApJS, 130, 1

Rengelink, R. B., Tang, Y., de Bruyn, A. G., Miley, G. K., Bremer, M. N., Röttgering, H. J. A., \& Bremer, M. A. R. 1997, A\&AS, 124, 259

Sargent, W. L. W., Steidel, C. C., \& Boksenberg, A. 1989, ApJS, 69, 703

Schneider, D. P., Schmidt, M., \& Gunn, J. E. 1991, AJ, 101, 2004

Snijders, M. A., Boksenberg, A., Penston, M. V., \& Sargent, W. L. 1982, MNRAS, 201, 801

Srianand, R., \& Petitjean, P. 1998, A\&A, 335, 33

Srianand, R., Petitjean, P., \& Ledoux, C. 2000, Nature, 408, 931

Steidel, C. C., Bowen, D. V., Blades, J. C., \& Dickenson, M. 1995, ApJ, 440, L45

Stepanian, J. A., Chavushian, V. H., Chaffee, F. H., Foltz, C. B., \& Green, R. F. 1996, A\&A, 309, 702

Stern, D., Djorgovski, S. G., Perley, R. A., de Carvalho, R. R., \& Wall, J. V. 2000, AJ, 119, 1526

Stickel, M., \& Kühr, H. 1996, A\&AS, 115, 1

Stocke, J. T., \& Rector, T. A., 1999, in Highly Redshifted Radio Lines, ASP Conf. Series 156, ed. C. L. Carilli, S. J. E. Radford, K. M. Menten, \& G. I. Langston (San Frcncisco: ASP), 183

Storrie-Lombardi, L. J., \& Wolfe, A. M. 2000, ApJ, 543, 552

Storrie-Lombardi, L. J., McMahon, R. G., Irwin, M. J., \& Hazard, C. 1996, ApJ, 468, 121
Teräsranta, H., et al. 1998, A\&AS, 132, 305

Tornikoski, M., Lainela, M., \& Valtaoja, E. 2000, AJ, 120, 2278

Turnshek, D. A., \& Bohlin, R. C. 1993, ApJ, 407, 60

Turnshek, D. A., Rao, S., Nestor, D., Lane, W., Monier, E., Bergeron, J., \& Smette, A. 2001, ApJ, 553, 288

Turnshek, D. A., Wolfe, A. M., Lanzetta, K. M., Briggs, F. H., Cohen, R. D., Foltz, C. B., Smith, H. E., \& Wilkes, B. J. 1989, ApJ, 344, 567

Tytler, D. 1982, Nature, 298, 427

Tytler, D. 1987a, ApJ, 321, 49

Tytler, D. 1987b, ApJ, 321, 69

Véron-Cetty, M. P., \& Véron, P. 2001, Quasars and Active Galactic Nuclei, 10th edition (Haute-Provence Observatory)

Visnovsky, K. L., Impey, C. D., Foltz, C. B., Hewett, P. C., Weymann, R. J., \& Morris, S. L. 1992, ApJ, 391, 560

Vladilo, G., Centurion, M., Falomo, R., \& Molaro, P. 1997, A\&A, 327,47

Wadadekar, Y., \& Kembhavi, A. 1999, AJ, 118, 1435

Wampler, E. J., Gaskell, C. M., Burke, W. L., \& Baldwin, J. A. 1984, ApJ, 276, 403

Wardle, J. F. C., \& Miley, G. K. 1971, ApJ, 164, L119

Webb, J. K., Carswell, R. F., Irwin, M. J., \& Penston, M. V. 1991, MNRAS, 250, 657

Webb, J. K., Flambaum, V. V., Churchill, C. W., Drinkwater, M. J., \& Barrow, J. D. 1999, PhRvL, 82, 884

Webb, J. K., Murphy, M. T., Flambaum, V. V., Dzuba, V. A., Barrow, J. D., Churchill, C. W., Prochaska, J. X., \& Wolfe, A. M. 2001, PhRvL, 87, 091301

White, R. L., \& Becker, R. H. 1992, ApJS, 79, 331

White, R. L., Kinney, A. L., \& Becker, R. H. 1993, ApJ, 407, 456

White, R. L., et al. 2000, ApJS, 126, 133

Wiklind, T., \& Combes, F. 1994a, A\&A, 286, L9

Wiklind, T., \& Combes, F. 1994b, A\&A, 288, L41

Wiklind, T., \& Combes, F. 1995, A\&A, 299, 382

Wiklind, T., \& Combes, F. 1996, Nature, 379, 139

Wolfe, A. M., \& Davis, M. M. 1979, AJ, 84, 699

Wolfe, A. M., Briggs, F. H., \& Davis, M. M. 1982, ApJ, 259, 495

Wolfe, A. M., Briggs, F. H., \& Jauncey, D. L. 1981, ApJ, 248,460

Wolfe, A. M., Broderick, J. J., Condon, J. J., \& Johnston, K. J. 1978, ApJ, 222, 752

Wolfe, A. M., Lanzetta, K. M., Foltz, C. B., \& Chaffee, F. H. 1995, ApJ, 454, 698

Wolfe, A. M., Turnshek, D. A., Smith, H. E., \& Cohen, R. D. 1986 , ApJS, 61, 249

Wolfe, A. M., Briggs, F. H., Turnshek, D. A., Davis, M. M., Smith, H. E., \& Cohen, R. D. 1985, ApJ, 294, L67

Wolfe, A. M., Fan, X., Tytler, D., Vogt, S. S., Keane, M. J., \& Lanzetta, K. M. 1994, ApJ, 435, L101

Wright, A., \& Otrupcek, R. 1990, Parkes Catalogue (Parkes: Australia Telescope National Facility)

Wright, A. E., Griffith, M. R., Burke, B. F., \& Ekers, R. D. 1994, ApJS, 91, 111

Wright, A. E., Griffith, M. R., Hunt, A. J., Troup, E., Burke, B. F., \& Ekers, R. D. 1996, ApJS, 103, 145

Zhang, F. J., Spencer, R. E., Schilizzi, R. T., Fanti, C., Bååth, L. B., \& Su, B. M. 1994, A\&A, 287, 32 